\title{
Un método de punto proximal escalarizado inexacto para minimización multiobjetivo cuasi-convexa en espacios Euclidianos
}

\author{
Erik Papa Quiroz ${ }^{1}$ y Segundo Cruzado Acuña ${ }^{2}$
}

Resumen: En este artículo presentamos un método de punto proximal escalarizado inexacto para resolver problemas irrestrictos de minimización multiobjetivo cuasiconvexas definidos en espacios Euclidianos, donde las funciones vectoriales son localmente Lipschitz. Bajo algunas hipótesis naturales, probamos que la sucesión generada por el método está bien definida, y converge globalmente. Seguidamente proporcionando al método propuesto dos criterios de error, se obtienen dos variantes del mismo, y se prueba que las sucesiones generadas por cada una de estas variantes, convergen hacia un punto crítico Pareto-Clarke del problema.

Palabras clave: Método de punto proximal; función cuasi-convexa; optimización multiobjetivo; subdiferencial de Clarke; subdiferencial de Fréchet.

\section{An inexact scalarization proximal point method for multiobjective quasiconvex minimization in Euclidean spaces}

\begin{abstract}
In this paper, we present an inexact scalarized proximal point method to solve unconstrained quasiconvex multiobjective minimization problems defined in Euclidean spaces, where the vector functions are locally Lipschitz. Under some natural assumptions, we prove that the sequence generated by the method is well defined and converges globally. Next, introduzing two error criteria on the method, two variants are obtained, and it is proved that the sequences generated by each one of these variants, converge to a Pareto-Clarke critical point of the problem.
\end{abstract}

Keywords: Proximal point method; quasiconvex function; multiobjetive optimization; Clarke subdifferential; Fréchet subdifferential.

Recibido: 18/12/2018. Aceptado: 02/03/2019. Publicado online: 13/05/2019.

(CLos autores. Este artículo es publicado por la Revista PESQUIMAT de la Facultad de Ciencias Matemáticas, Universidad Nacional Mayor de San Marcos. Este es un artículo de acceso abierto, distribuido bajo los términos de la licencia Creative Commons Atribucion-No Comercia-Compartir Igual 4.0 Internacional.(http://creativecommons.org/licenses/by-nc-sa/4.0/) que permite el uso no comercial, distribución y reproducción en cualquier medio, siempre que la obra original sea debidamente citada. Para información, por favor póngase en contacto con revistapesquimat.matematica@unmsm.edu.pe

\footnotetext{
${ }^{1}$ UNMSM, Facultad de Ciencias Matemáticas. e-mail: epapaq@unmsm.edu.pe

${ }^{2}$ UNMSM, Facultad de Ciencias Matemáticas, e-mail: scruzadoa@gmail.com
} 


\section{Introducción}

En el presente artículo, se tiene como objetivo resolver el siguiente problema irrestricto de minimización multiobjetivo cuasi-convexa, definido en espacios Euclidianos

$$
\min _{x \in \mathbb{R}^{n}} F(x)=\min \left\{F(x), x \in \mathbb{R}^{n}\right\} .
$$

Decimos que que el problema anterior es multiobjetivo, debido a que $F: \mathbb{R}^{n} \rightarrow \mathbb{R}^{p}$, es de la forma $F(x)=\left(F_{1}(x), \cdots, F_{p}(x)\right), p \geq 2 ;$ y cuasi-convexa, dado que cada una de las componentes de la función $F\left(F_{i}: \mathbb{R}^{n} \rightarrow \mathbb{R}, i=1,2, \cdots, p\right)$ satisfacen la siguiente desigualdad:

$$
F_{i}(\lambda x+(1-\lambda) y) \leq \max \left\{F_{i}(x), F_{i}(y)\right\}, \forall \lambda \in[0,1], \forall x, y \in \mathbb{R}^{n}, \mathrm{y} \forall i \in\{1,2, \cdots, p\} .
$$

La elección de trabajar con funciones multiobjetivo se justifica en el hecho de que en la vida diaria, muchas veces para tomar una decisión, se debe optimizar muchas funciones objetivo a la vez. Por ejemplo en el proceso de compra de una casa, uno quiere la "mejor", al mejor precio, pero ¿qué es lo mejor?. En base al sentido común es razonable aceptar como "mejor casa", aquella que es la más amplia, la que resulte más confortable, la que tenga mejor ubicación, y ciertamente aquella con mínimos gastos de mantenimiento; si a estas funciones objetivo le agregamos el costo de la casa (el cual queremos minimizar), entonces tendríamos una función con cinco objetivos, o en general una función multiobjetivo, en cambio si solamente consideramos uno de los objetivos, entonces estaríamos ante un problema de optimización simple, o mono-objetivo, estudiado en la optimización clásica [5, p. 151].

Por otro lado, la elección de las funciones cuasi-convexas, no solo se debe a la reciente atención que éstas vienen recibiendo por una parte de la comunidad científica, si no también a la amplia gama de aplicaciones, por ejemplo en teoría de localización (en la cuál sus modelos aparecen representados por función objetivo cuasi-convexas), ver [16], en programación fraccional, y especialmente en teoría de decisión del consumidor en económica, en la cuál la minimización cuasi-convexa está relacionado a las preferencias convexas, ver por ejemplo [19]. Algunos artículos relacionados a ellos son los siguientes: [18], [29], [25], entre otros.

Por lo tanto, para resolver el problema (1), donde $F$ es una función localmente Lipschitz, y además satisface algunas otras condiciones, presentaremos un Método de Punto Proximal Escalarizado Inexacto (MPPEI) para minimización multiobjetivo cuasi-convexa, el cuál tiene el siguiente proceso iterativo: dado un $x^{k} \in \mathbb{R}^{n}$, con $k=0,1,2, \cdots$, encontrar un $x^{k+1} \in \Omega_{k}$, tal que:

$$
e^{k+1} \in \partial^{0}\left(\left\langle F(.), z_{k}\right\rangle+\frac{\alpha_{k}}{2}\left\|\cdot-x^{k}\right\|^{2}\right)\left(x^{k+1}\right)+\mathcal{N}_{\Omega_{k}}\left(x^{k+1}\right),
$$

donde $\partial^{0}$ es el subdiferencial de Clarke (ver Definición 2.2.2), $\Omega_{k}=\left\{x \in \mathbb{R}^{n}: F(x) \preceq{ }^{1} F\left(x^{k}\right)\right\} \subset$ $\mathbb{R}^{n}, \alpha_{k}>0,\left\{z_{k}\right\} \subset \mathbb{R}_{\geq}^{p} \backslash\{0\}=\left\{y \in \mathbb{R}^{p}: y_{i} \geq 0 ; ; i=1, \cdots, p ; y \neq 0\right\},\left\|z_{k}\right\|=1, \mathrm{y}$ $N_{\Omega_{k}}\left(x^{k+1}\right)=\left\{d \in \mathbb{R}^{n}:\left\langle\bar{d}, x-x^{k+1}\right\rangle \leq 0, \forall x \in \Omega_{k}\right\}$ es el cono normal a $\Omega_{k}$ en $x^{k+1}$.

El MPPEI anteriormente indicado, representa una extensión hacia su versión inexacta del Método de Punto Proximal Escalarizado (MPPE) presentado por [1], quienes trabajando con hipótesis semejantes, y considerando en (2) que $e^{k+1}=0$, probaron que la sucesión $\left\{x^{k+1}\right\}$ generada por el método está bien definida, y además obtienen la convergencia global a un punto crítico Pareto-Clarke, y cuando $F$ es convexa, obtienen la convergencia a una solución débil de Pareto.

\footnotetext{
${ }^{1}$ Decimos que $F(x) \preceq F\left(x^{k}\right)$, para $F(x), F\left(x^{k}\right) \in \mathbb{R}^{p}$, si y solamente si $F_{i}(x) \leq F_{i}\left(x^{k}\right)$ para todo $i=1, \cdots, p$.
} 
La extensión hacia su versión inexacta del método propuesto por [1], responde a la necesidad de construir algoritmos de punto proximal computacionalmente más implementables, y se justifica en la dificultad que por lo general se tiene para encontrar un valor $x^{k}$ que resuelva de forma exacta los subproblemas generados por el Método del Punto Proximal. En ese sentido se consideran errores de aproximación $e^{k}$, los cuales al cumplir determinadas propiedades, permiten generalizar las propiedades de convergencia de los algoritmos exactos, ver por ejemplo los artículos: [25] y [24], en el último de los cuáles los autores presentan una versión inexacta del primero de ellos (trabajando en ambos casos con funciones cuasi-convexas semi-continuas inferiores). Otros trabajos para funciones cuasi-convexas diferenciables pero que hacen uso de iteraciones exactas también fueron desarrollados por [22], [11], [14] y [29].

Actualmente, en el campo de las variedades de Hadamard (variedades Riemannianas con curvatura seccional no positiva), la versión inexacta del método propuesto por [1], está siendo estudiada (ver por ejemplo [3]), sin embargo aún no existen publicaciones al respecto. Por lo tanto, al no existir en los espacios Euclidianos trabajos teóricos relacionados con la extensión de dicho método hacia su versión inexacta, en el presente artículo realizamos dicho trabajo. Además, siguiendo un enfoque semejante al realizado por [4] (quienes trabajaron con funciones mono-objetivo definidas en espacios de Hadamard), presentamos y analizamos los resultados de convergencia de dos variantes del algoritmo MPPEI, dados por los siguientes criterios de error:

$$
\begin{aligned}
\sum_{k=0}^{+\infty} \frac{\left\|e^{k+1}\right\|}{\alpha_{k}} & <+\infty \\
\frac{\left\|e^{k+1}\right\|}{\alpha_{k}} & \leq \eta_{k}\left\|x^{k+1}-x^{k}\right\|, \text { donde } \sum_{k=0}^{+\infty} \eta_{k}^{2}<+\infty
\end{aligned}
$$

el primero de los cuáles es una versión clásica introducida por [27], y el segundo de ellos, fue estudiado por [30]. También, bajo ciertas hipótesis (como por ejemplo la acotación del parámetro $\alpha_{k}$ ), probamos que la sucesión $\left\{x^{k}\right\}$ generada por cada una de estas variantes converge hacia algún punto crítico Pareto-Clarke (ver Definición 2.4.1) del problema (1); y si $F$ es convexa, probamos que el punto de convergencia de $\left\{x^{k}\right\}$, es una solución débil de Pareto del problema (1).

El resto del documento está organizado de la siguiente manera: En la Sección 2, presentamos algunas definiciones y resultados relacionados con las cuasi-convexidad de funciones localmente Lipschitz, con los subdiferenciales de Fréchet y Clarke, y con la teoría de optimización multiobjetivo, necesaria para los fines del presente artículo. En la Sección 3, presentamos el problema de optimización y el algoritmo inexacto MPPEI. Finalmente, en la sección 4, proporcionando a dicho algoritmo de dos criterios de error, obtenemos dos variantes del mismo (MPPEI1 y MPPEI2), y probamos que la sucesiones generadas por cada una de estas variantes, convergen hacia un punto crítico Pareto-Clarke del problema. Cabe resaltar que el análisis de la velocidad de convergencia del algoritmo MPPEI2, recientemente ha sido estudiado por [?], por lo que se recomienda revisar dicho artículo, dado que constituye un complemento del presente trabajo.

\section{Preliminares}

Los conceptos y resultados que presentamos en esta sección, han sido tomados de [7], [31], [21] y [28], y son de fundamental importancia para el desarrollo del presente artículo, a lo largo del cual $\mathbb{R}^{n}$ denota el espacio Euclidiano, quien junto con el producto interno canónico $\langle x, y\rangle=\sum_{i=1}^{n} x_{i} y_{i}$ y la norma en $x$ dada por $\|x\|=\sqrt{\langle x, x\rangle}$, representa un espacio vectorial. 


\subsection{Definiciones, notaciones y algunos resultados básicos}

Dada una función con valores en los reales extendidos $f: \mathbb{R}^{n} \rightarrow \mathbb{R} \cup\{+\infty\}$, denotamos por $\operatorname{dom}(f)=\left\{x \in \mathbb{R}^{n}: f(x)<+\infty\right\}$, al dominio efectivo de $f$. Decimos que $f$ es propia, si $\operatorname{dom}(f) \neq \emptyset$ y $\forall x \in \operatorname{dom}(f)$ se tiene que $f(x)>-\infty$. Además, $f$ es semicontinua inferior (sci) en $\bar{x} \in \mathbb{R}^{n}$ si para toda sucesión $\left\{x_{k}\right\}_{k \in \mathbb{N}}$ convergente a $\bar{x}$ se tiene que $f(\bar{x}) \leq \liminf _{k \rightarrow+\infty} f\left(x_{k}\right)$; si $f$ es sci para todo $x \in \mathbb{R}^{n}$, entonces decimos que $f$ es sci en $\mathbb{R}^{n}$, o simplemente que $f$ es sci. Si $\lim _{\|x\| \rightarrow+\infty} f(x)=+\infty, f$ es llamada coerciva.

Definición 2.1.1 Sea $f: \mathbb{R}^{n} \rightarrow \mathbb{R} \cup\{+\infty\}$ una función propia. Decimos que $f$ es localmente Lipschitz en $x \in \operatorname{dom}(f)$, si existe algún $\epsilon_{x}>0$ tal que:

$$
|f(z)-f(y)| \leq L_{x}\|z-y\| \quad \forall z, y \in B\left(x, \epsilon_{x}\right),
$$

donde $B\left(x, \epsilon_{x}\right)=\left\{y \in \mathbb{R}^{n},\|y-x\|<\epsilon_{x}\right\}$ y $L_{x}>0$ es la constante de Lipschitz. Decimos que $f$ es localmente Lipschitz en $\mathbb{R}^{n}$, si f es localmente Lipschitz en cada $x \in \mathbb{R}^{n}$.

Definición 2.1.2 Sean $C \subset \mathbb{R}^{n} y \bar{x} \in C$. El cono normal al punto $\bar{x}$ relacionado al conjunto $C$ está dado por $N_{C}(\bar{x})=\left\{d \in \mathbb{R}^{n}:\langle d, x-\bar{x}\rangle \leq 0, \forall x \in C\right\}$.

Proposición 2.1.1 Si $f: \mathbb{R}^{n} \rightarrow \mathbb{R} \cup\{+\infty\}$ es una función propia, semicontinua inferior $y$ coerciva, entonces el valor óptimo $f^{*}$ es finito, y además el conjunto arg $\min \left\{f(x): x \in \mathbb{R}^{n}\right\}$ es no vacío y compacto.

Demostración. Véase [28], Teorema 1.9.

Teorema 2.1.1 Si una función $f: \mathbb{R}^{n} \supseteq X \rightarrow \mathbb{R} \cup\{+\infty\}$ es coerciva y semicontinua inferior en un conjunto $X \neq \emptyset$, entonces existe un punto que representa un mínimo global de $f$ en $X$.

Demostración. Véase [23], Corolario 3.4.

Definición 2.1.3 Sea $f: \mathbb{R}^{n} \rightarrow \mathbb{R} \cup\{+\infty\}$ una función propia. Decimos que $f$ es cuasi-convexa si $f(\alpha x+(1-\alpha) y) \leq \max \{f(x), f(y)\}$ para todo $x, y \in \mathbb{R}^{n}$ y para todo $\alpha \in[0,1]$.

Definición 2.1.4 Sea $F: \mathbb{R}^{n} \rightarrow \mathbb{R}^{p}$ (donde $F_{i}: \mathbb{R}^{n} \rightarrow \mathbb{R}$ ) una función. Decimos que $F$ es

1. $\mathbb{R}_{\geqq}^{p}$-cuasi-convexa si y sólo si cada componente de $F$ es cuasi-convexa;

2. $\mathbb{R}_{\geqq}^{p}$-convexa si cada componente de $F$ es convexa;

3. locamente Lipschitz en $\mathbb{R}^{n}$ si cada componente de F es locamente Lipschitz en $\mathbb{R}^{n}$.

El resultado que presentamos a continuación será usado para asegurar que la sucesión $\left\{x^{k}\right\}$ generada por cada una de las variantes del algoritmo MPPEI (MPPEI1 y MPPEI2), es acotada.

Lema 2.1.1 Sean $\left\{v_{k}\right\},\left\{\lambda_{k}\right\}$ y $\left\{\beta_{k}\right\}$ sucesiones no negativas de números reales que satisfacen

$$
v_{k} \leq\left(1+\lambda_{k}\right) v_{k-1}+\beta_{k},
$$

donde $\sum_{k=1}^{+\infty} \beta_{k}<+\infty, \sum_{k=1}^{+\infty} \lambda_{k}<+\infty$. Entonces la sucesión $\left\{v_{k}\right\}$ es convergente.

Demostración. Véase [26], Lema 2, página 44. 


\subsection{Subdiferenciales Generalizados}

Los conceptos y resultados que presentamos en la presente subsección, fueron tomados de $[13,12]$ y $[28$, cap. 8 y 10$]$.

Si bien la mayoría de los conceptos que siguen pueden ser desarrollados para funciones del tipo $f: X \rightarrow Y$, donde $X, Y$ son espacios de Banach, en la presente solamente nos limitaremos a los espacios Euclidianos y a funciones del tipo $f: \mathbb{R}^{n} \rightarrow \mathbb{R} \cup\{+\infty\}$.

\subsubsection{Subdiferencial de Fréchet y en el límite}

Definición 2.2.1 Sea $f: \mathbb{R}^{n} \rightarrow \mathbb{R} \cup\{+\infty\}$ una función propia.

1. Para cada $x \in \operatorname{dom}(f)$, donde $f(x)$ es finito, el conjunto de subgradientes regulares (también llamado subdiferencial de Fréchet) de $f$ en $x$, denotado por $\widehat{\partial} f(x)$ esta formado por un conjunto de vectores $d \in \mathbb{R}^{n}$, tales que

$$
f(y) \geq f(x)+\langle d, y-x\rangle+\theta(\|y-x\|), \text { donde } \lim _{\substack{y \rightarrow x \\ y \neq x}} \frac{\theta(\|y-x\|)}{\|y-x\|}=0,
$$

o equivalentemente, $\widehat{\partial} f(x)=\left\{d \in \mathbb{R}^{n}: \liminf _{\substack{y \rightarrow x \\ y \neq x}} \frac{f(y)-f(x)-\langle d, y-x\rangle}{\|y-x\|} \geq 0\right\}$.

Si $x \notin \operatorname{dom}(f)$ entonces $\widehat{\partial} f(x)=\emptyset$.

2. El conjunto de subgradientes generalizados (también llamado subdiferencial en el límite) de $f$ en $x$, denotado por $\partial^{\text {Lim }} f(x)$ esta definido por

$$
\partial^{L i m} f(x)=\left\{d \in \mathbb{R}^{n}: \exists x^{l} \rightarrow x, f\left(x^{l}\right) \rightarrow f(x), d^{l} \in \widehat{\partial} f\left(x^{l}\right) y d^{l} \rightarrow d\right\} .
$$

Proposición 2.2.1 Para una función $f: \mathbb{R}^{n} \rightarrow \mathbb{R} \cup\{+\infty\}$ y un punto $\bar{x} \in \operatorname{dom}(f)$ en el cuál $f$ es finito, los conjuntos subgradientes $\partial^{\text {Lim }} f(\bar{x})$ y $\widehat{\partial} f(\bar{x})$ son cerrados, con $\widehat{\partial} f(\bar{x})$ convexo y $\widehat{\partial} f(\bar{x}) \subset \partial^{\operatorname{Lim}} f(\bar{x})$.

Demostración. Véase [28], Teorema 8.6.

Proposición 2.2.2 (Regla generalizada de Fermat). Si una función propia $f: \mathbb{R}^{n} \rightarrow \mathbb{R} \cup\{+\infty\}$ tiene un minimo local en $\bar{x} \in \operatorname{dom}(f)$, entonces $0 \in \widehat{\partial} f(\bar{x})$.

Demostración. Véase [28], Teorema 10.1.

Proposición 2.2.3 Sea $C \subset \mathbb{R}^{n}, C \neq \emptyset$, un conjunto convexo cerrado y $x \in C$, entonces

$$
\partial^{\operatorname{Lim}}\left(\delta_{C}\right)(x)=N_{C}(x)
$$

Demostración. La demostración es una implicación directa del Ejercicio 4.5 (página 61) y la Proposición 2.9 (página 30) de [13].

Proposición 2.2.4 Sean $f, g: \mathbb{R}^{n} \rightarrow \mathbb{R} \cup\{+\infty\}$ funciones propias, tales que $f$ es localmente Lipschitz en $\bar{x} \in \operatorname{dom}(f) \cap \operatorname{dom}(g)$, y g es semicontinua inferior en dicho punto, entonces

$$
\partial^{\operatorname{Lim}}(f+g)(\bar{x}) \subset \partial^{\operatorname{Lim}} f(\bar{x})+\partial^{\operatorname{Lim}} g(\bar{x}) .
$$

Demostración. Véase [21], Teorema 2.33. 


\subsubsection{Subdiferencial de Clarke}

Definición 2.2.2 Sean $f: \mathbb{R}^{n} \rightarrow \mathbb{R} \cup\{+\infty\}$ una función propia y localmente Lipschitz en $x \in \operatorname{dom}(f), y d \in \mathbb{R}^{n}$. La derivada direccional de Clarke de $f$ en $x$ en la dirección $d$, denotada por $f^{0}(x, d)$, es definida por

$$
f^{0}(x, d)=\limsup _{\substack{y \rightarrow x \\ t \downarrow 0}} \frac{f(y+t d)-f(y)}{t},
$$

y el subdiferencial de Clarke de $f$ en $x$, denotado por $\partial^{0} f(x)$ está definido por

$$
\partial^{0} f(x)=\left\{w \in \mathbb{R}^{n}:\langle w, d\rangle \leq f^{0}(x, d), \quad \forall d \in \mathbb{R}^{n}\right\} .
$$

Observación 2.2.1 De la definición anterior se sigue directamente que para todo $x \in \operatorname{dom}(f)$ se tiene que $\widehat{\partial} f(x) \subset \partial^{\text {Lim }} f(x) \subset \partial^{0} f(x)$ [8, inclusión (7)].

Lema 2.2.1 Sean $f, g: \mathbb{R}^{n} \rightarrow \mathbb{R} \cup\{+\infty\}$ funciones propias y localmente Lipschitz en $x \in$ $\operatorname{dom}(f) \cap \operatorname{dom}(g)$. Luego para todo $d \in \mathbb{R}^{n}$ se tiene que

i) $(f+g)^{0}(x, d) \leq f^{0}(x, d)+g^{0}(x, d)$;

ii) $(\lambda f)^{0}(x, d)=\lambda f^{0}(x, d)$, para todo $\lambda \geq 0$;

iii) $f^{0}(x, \lambda d)=\lambda f^{0}(x, d)$, para todo $\lambda \geq 0$.

Demostración. Es inmediata, y se sigue directamente de la Definición 2.2.2.

Lema 2.2.2 Si $f: \mathbb{R}^{n} \rightarrow \mathbb{R} \cup\{+\infty\}$ es una función propia y localmente Lipschitz en $x \in \operatorname{dom}(f)$, y $\lambda$ un escalar arbitrario, entonces:

$$
\partial^{0}(\lambda f)(x)=\lambda \partial^{0} f(x) .
$$

Demostración. Véase [12], Proposición 2.3.1.

Lema 2.2.3 Sean $f_{i}: \mathbb{R}^{n} \rightarrow \mathbb{R} \cup\{+\infty\}, i=1, \cdots, p$, funciones propias y localmente Lipschitz en $x \in \cap_{i=1}^{p} \operatorname{dom}\left(f_{i}\right)$, entonces

$$
\partial^{0}\left(\sum_{i=1}^{p} f_{i}\right)(x) \subset \sum_{i=1}^{p} \partial^{0} f_{i}(x) .
$$

Demostración. La prueba se sigue inmediatamente a partir del Lema 2.2.1(i).

Proposición 2.2.5 Si $f: \mathbb{R}^{n} \rightarrow \mathbb{R} \cup\{+\infty\}$ es una función propia y localmente Lipschitz de constante $K>0$ cerca de $x$, entonces $f^{0}$ es semicontinua superior (scs), es decir si $(x, d) \in$ $\mathbb{R}^{n} \times \mathbb{R}^{n} y\left\{\left(x^{k}, d^{k}\right)\right\}$ es una sucesión en $\mathbb{R}^{n} \times \mathbb{R}^{n}$ tal que $\lim _{k \rightarrow+\infty}\left(x^{k}, d^{k}\right)=(x, d)$, entonces

$$
\limsup _{k \rightarrow+\infty} f^{0}\left(x^{k}, d^{k}\right) \leq f^{0}(x, d)
$$

Demostración. Véase [12], Proposición 2.1.1. 
Proposición 2.2.6 Sea $f: \mathbb{R}^{n} \rightarrow \mathbb{R} \cup\{+\infty\}$ una función propia, cuasi-convexa y localmente Lipschitz en $\mathbb{R}^{n}$. Si $g \in \partial^{0} f(x)$ y $f(y)<f(x)$, entonces $\langle g, y-x\rangle \leq 0$.

Demostración. Véase [2], Teorema 2.1.

Proposición 2.2.7 Si $f: \mathbb{R}^{n} \rightarrow \mathbb{R} \cup\{+\infty\}$ es una función convexa, propia, y localmente Lipschitz de constante $K>0$ en $x \in \mathbb{R}^{n}$, entonces $\partial^{0} f(x)$ coincide con el subdiferencial en $x$ (en el sentido del análisis convexo), y $f^{0}(x, d)$ coincide con la derivada direccional $f^{\prime}(x, d)$ para cadad.

Demostración. Véase [12], Proposición 2.2.7.

Teorema 2.2.1 Si $f: \mathbb{R}^{n} \rightarrow \mathbb{R}$ es una función localmente Lipschitz y continumente diferenciable, entonces $f^{0}(x, d)$ coincide con la derivada direccional $f^{\prime}(x, d)$ para cada $d$.

Demostración. Véase [13], Teorema 10.8, página 197.

Lema 2.2.4 Sean $f: \mathbb{R}^{n} \rightarrow \mathbb{R} \cup\{+\infty\}$ una función localmente Lipschitz, y $g: \mathbb{R}^{n} \rightarrow \mathbb{R}$ una función convexa, entonces:

$$
(f+g)^{0}(x, d)=f^{0}(x, d)+g^{0}(x, d) \quad \forall x, d \in \mathbb{R}^{n} .
$$

Además, si g es diferenciable se tiene que

$$
\partial^{0}(f+g)(x)=\partial^{0} f(x)+\nabla g(x) \quad \forall x \in \mathbb{R}^{n} .
$$

Demostración. Véase [6], Lema 3.1, página 8.

\subsection{Teoría de optimización multiobjetivo}

Las definiciones, propiedades y notaciones que presentamos en esta subsección han sido tomadas de [17] y [20], así que para mayores detalles se recomienda revisar dichas fuentes.

A lo largo de este artículo vamos a considerar el cono $\mathbb{R}_{\geq}^{p}=\left\{y \in \mathbb{R}^{p}: y_{i} \geq 0, \forall i=1, \cdots, p\right\}$, el cuál induce un orden parcial $\preceq$ en $\mathbb{R}^{p}$, que indica que $y \preceq y^{\prime}$, para $y, y^{\prime} \in \mathbb{R}^{p}$, si y solo sí $y^{\prime}-y \in \mathbb{R}_{\geqq}^{p}$, lo cual significa que $y_{i} \leq y_{i}^{\prime}$ para todo $i=1,2, \cdots, p$. Considerando la relación anterior, definimos el cono $\mathbb{R}_{>}^{p}=\left\{y \in \mathbb{R}^{p}: y_{i}>0, \forall i=1, \cdots, p\right\}$, el cuál induce el orden $\prec$ en $\mathbb{R}^{p}$, que indica que $y \prec y^{\prime}$, para $y, y^{\prime} \in \mathbb{R}^{p}$, si y solo sí $y^{\prime}-y \in \mathbb{R}_{>}^{p}$, lo cual significa que $y_{i}<y_{i}^{\prime}$ para todo $i=1,2, \cdots, p$. Esos órdenes parciales establecer una clase de problemas conocidos en la literatura como problemas de optimización multiobjetivo $\left(P_{S} O M\right)$, los cuáles en su versión irrestricta (objeto de estudio del presente artículo) están formulados de la siguiente manera:

$$
\min _{x \in \mathbb{R}^{n}} F(x)=\min \left\{F(x): x \in \mathbb{R}^{n}\right\} .
$$

donde $F: \mathbb{R}^{n} \rightarrow \mathbb{R}^{p}$, con $F(x)=\left(F_{1}(x), \ldots, F_{p}(x)\right), p \geq 2$, denota el vector de funciones objetivo.

Definición 2.3.1 [17, Definición 2.1]. Decimos que un punto $\hat{x} \in \mathbb{R}^{n}$ es una solución de Pareto (también conocida como solución eficiente, óptimo de Pareto, solución no dominada, o solución no interior) del problema (4), si no existe otra $x \in X$ tal que $F_{i}(x) \leq F_{i}(\hat{x})$ para todo $i=1, \ldots, p$ y $F_{j}(x)<F_{j}(\hat{x})$ para al menos un indice $j \in\{1, \ldots, p\}$. 
Definición 2.3.2 [17, Definición 2.24]. Un punto $\hat{x} \in \mathbb{R}^{n}$ es una solución débil de Pareto (solución débilmente eficiente o débilmente óptima de Pareto) del problema (4), si no existe $x \in X$ tal que $F(x)<F(\hat{x})$, es decir $F_{i}(x)<F_{i}(\hat{x})$ para todo $i=1, \cdots, p$.

En base a las Definiciones 2.3.1 y 2.3.2 vamos a considerar a $X_{E}\left(X_{w E}\right)$, como el conjunto de soluciones eficientes (débilmente eficientes) del problema (4); además, en función de dichas definiciones, fácilmente se puede verificar que $X_{E} \subset X_{w E}$.

\subsection{Direcciones de descenso}

Ahora podemos introducir la definición de punto crítico Pareto-Clarke para funciones localmente Lipschitz en $\mathbb{R}^{n}$, definición que jugará un papel clave en el desarrollo de la presente artículo.

Definición 2.4.1 [15, Definición 4.6]. Sea $F: \mathbb{R}^{n} \rightarrow \mathbb{R}^{p}$ una función localmente Lipschitz en $\mathbb{R}^{n}$. Decimos que $\hat{x} \in \mathbb{R}^{n}$ es un punto crítico Pareto-Clarke de $F$ si para todas las direcciones $d \in \mathbb{R}^{n}$, existe $i_{0}=i_{0}(d) \in\{1, \cdots, p\}$ tal que $F_{i_{0}}^{0}(\hat{x}, d) \geq 0$.

La definición anterior esencialmente nos dice que en $\mathbb{R}^{n}$ no existe ninguna dirección de descenso para todas las funciones objetivo.

Observación 2.4.1 De la Definición 2.4.1 se sigue que si un punto $x$ no es un punto crítico Pareto-Clarke, entonces existe una dirección $d \in \mathbb{R}^{n}$ que satisface

$$
F_{i}^{0}(x, d)<0 \quad \forall i \in\{1, \cdots, p\},
$$

lo cual implica que para cada $i \in\{1, \cdots, p\}$, d es una dirección de descenso para cada función $F_{i}$, es decir existe un $\epsilon>0$, tal que $F_{i}(x+t d)<F_{i}(x)$ para todo $t \in(0, \epsilon]$ y para todo $i \in\{1, \cdots, p\}$. Lo cual en función de las componentes de orden de la función multiobjetivo significa que

$$
F(x+t d) \prec F(x) \quad \forall t \in(0, \epsilon] .
$$

Proposición 2.4.1 Sea $\hat{x}$ un punto crítico Pareto-Clarke de una función localmente Lipschitz $F: \mathbb{R}^{n} \rightarrow \mathbb{R}^{p}$. Si F es $\mathbb{R}_{\geqq}^{p}$-convexa, entonces $\hat{x}$ es una solución débil de Pareto del problema (4).

Demostración. Véase [1], Proposición 2.6.1.

\section{Definición del problema y el algoritmo MPPEI}

Estamos interesados en resolver el problema de minimización multiobjetivo (4), dado por:

$$
\min _{x \in \mathbb{R}^{n}} F(x)=\min \left\{F(x), x \in \mathbb{R}^{n}\right\},
$$

donde $F: \mathbb{R}^{n} \longrightarrow \mathbb{R}^{p}$ satisface las siguientes hipótesis:

(H1) $0 \preceq F$, es decir que $F_{i}(x) \geq 0$ para todo $i \in\{1, \cdots, p\}$ y $x \in \operatorname{dom}\left(F_{i}\right)$, 
(H2) $F$ es localmente Lipschitz en $\mathbb{R}^{n}$ (ver Definición 2.1.1 y 2.1.4(3)),

(H3) $F$ es $\mathbb{R}_{\geq}^{p}$-cuasi-convexa (ver Definición 2.1.4(1)).

\subsection{El algoritmo}

Para resolver el problema (6), en esta subsección proponemos el Método de Punto Proximal Escalarizado Inexacto (MPPEI) con regularización cuadrática definido por:

Algorithm 1*

Algoritmo MPPEI Inicio: Escoger un punto inicial arbitrario

$$
x^{0} \in \mathbb{R}^{n} .
$$

Paso iterativo: Para $k=0,1, \cdots$, y dado un $x^{k}$, encontrar $x^{k+1} \in \Omega_{k}$ y $e^{k+1}$ tal que:

$$
e^{k+1} \in \partial^{0}\left(\left\langle F(.), z_{k}\right\rangle+\frac{\alpha_{k}}{2}\left\|.-x^{k}\right\|^{2}\right)\left(x^{k+1}\right)+\mathcal{N}_{\Omega_{k}}\left(x^{k+1}\right),
$$

donde $\Omega_{k}=\left\{x \in \mathbb{R}^{n}: F(x) \preceq F\left(x^{k}\right)\right\}, \alpha_{k}>0,\left\{z_{k}\right\} \subset \mathbb{R}_{\geqq}^{p} \backslash\{0\}$ y $\left\|z_{k}\right\|=1$.

Criterio de parada: Si $x^{k+1}=x^{k}$ o si $x^{k+1}$ es un punto crítico Pareto-Clarke (ver Definición 2.4.1), detener el algoritmo. En otro caso hacer $k \leftarrow k+1$ y retornar al paso iterativo.

Observación 3.1.1 1 . Si $e^{k+1}=0$ para todo $k \in \mathbb{N}$, entonces en la expresión (8) estaremos ante la versión exacta del Método de Punto Proximal Escalarizado (MPPE), propuesto por [1]. Así, se puede decir que el algoritmo que se está proponiendo representa una extensión a la versión inexacta del MPPE.

Además, si $F$ is $\mathbb{R}_{\geqq-}^{p}$ convexa, entonces la expresión (8) resulta en

$$
x^{k+1}=\operatorname{argmin}\left\{\left\langle F(.), z_{k}\right\rangle+\frac{\alpha_{k}}{2}\left\|\cdot-x^{k}\right\|^{2}: \quad x \in \Omega_{k}\right\},
$$

iteración que ha sido estudiada por [9], la cual fue tomada como referencia para la realización del MPPE (considerando en este caso funciones cuasi-convexas).

2. Si $F: \mathbb{R}^{n} \longrightarrow \mathbb{R}, z_{k}=1, \partial^{0}=\widehat{\partial}$ (donde $\widehat{\partial}$ representa el subdiferencial de Fréchet) $y$ $x^{k+1} \in \operatorname{int}\left(\Omega_{k}\right)$, entonces en (8) tendremos la siguiente iteración

$$
e^{k+1} \in \widehat{\partial}\left(f(.)+\frac{\alpha_{k}}{2}\left\|.-x^{k}\right\|^{2}\right)\left(x^{k+1}\right),
$$

estudiada por [24]. Además, si en en la expresión anterior hacemos que $e^{k+1}=0$, y consideramos algunas hipótesis adicionales, como que $x \geq 0$, obtendremos el algoritmo estudiado por [25].

\subsection{Existencia de los iterados}

Teorema 3.2.1 Si $F: \mathbb{R}^{n} \rightarrow \mathbb{R}^{p}$ es una función que satisface las hipótesis (H1), (H2) y (H3), entonces la sucesión $\left\{x^{k}\right\}$ generada por el algoritmo MPPEI está bien definida. 
Demostración. La demostración se sigue de [1], Teorema 3.2.1, considerando al final de dicha prueba que $e^{k+1}=0$.

Como estamos interesados en la convergencia asintótica del algoritmo MPPEI, a lo largo de este trabajo vamos a asumir que en cada iteración $x^{k+1}$ no es un punto crítico Pareto-Clarke, lo cual en función de la Observación 2.4.1-relación (5) va a implicar que $\operatorname{int}\left(\Omega_{k+1}\right) \neq \emptyset$. También vamos a asumir que (en cada iteración) $x^{k+1} \neq x^{k}$; si esta condición no es satisfecha, es decir si existe un $k_{0}$ tal que $x^{k_{0}+1}=x^{k_{0}}$, entonces de forma análoga a lo realizado en los Teoremas 4.1.1 y 4.2.1 (considerando las propiedades que éstos requieren), fácilmente se puede probar que $x^{k_{0}+1}$ es un punto crítico Pareto-Clarke de $F$.

A continuación presentamos otra hipótesis sobre la función $F$ y el punto inicial $x^{0}$, la cual está citada en varios trabajos concernientes a los algoritmos proximales. Ver por ejemplo [9], [?] y [?]. El conjunto

(H4) $\left(F\left(x^{0}\right)-\mathbb{R}_{\geq}^{p}\right) \cap F\left(\mathbb{R}^{n}\right)$ es $\mathbb{R}_{\geq}^{p}$-completo, lo cual significa que para toda sucesión $\left\{a^{k}\right\} \subset \mathbb{R}^{n}$, con $a^{0}=x^{0}$, tal que $F\left(a^{k+1}\right) \preceq F\left(a^{k}\right)$ para todo $k \in \mathbb{N}$, existe $a \in \mathbb{R}^{n}$ tal que $F(a) \preceq F\left(a^{k}\right)$ para todo $k \in \mathbb{N}$.

Observar que por las hipótesis $(\mathbf{H 1}),(\mathbf{H 2}),(\mathbf{H} 3)$ y $(\mathbf{H} 4)$, el conjunto $E$, dado por

$$
E=\left\{x \in \mathbb{R}^{n}: F(x) \preceq F\left(x^{k}\right), \forall k \in \mathbb{N}\right\},
$$

es un conjunto no vacío ${ }^{2}$, cerrado y convexo. Además, $E \subseteq \Omega_{k}$, para cualquier $k \in \mathbb{N}$.

\subsection{Resultados de convergencia}

El siguiente resultado jugará un papel esencial en el desarrollo del presente artículo. Más precisamente será útil en el análisis de convergencia de la sucesión generada por el algoritmo MPPEI.

Lema 3.3.1 Sea $F: \mathbb{R}^{n} \rightarrow \mathbb{R}^{p}$ una función que satisface las hipótesis (H1), (H2) y (H4), y $\left\{x^{k}\right\}$ la sucesión generada por el algoritmo MPPEI. Si $\hat{x}$ es un punto de acumulación de $\left\{x^{k}\right\}$, entonces $\hat{x} \in E$.

Demostración. Sea $\hat{x} \in \mathbb{R}^{n}$ un punto de acumulación de $\left\{x^{k}\right\}$, entonces existe una subsucesión $\left\{x^{k_{j}}\right\} \subseteq\left\{x^{k}\right\}$ tal que $\lim _{j \rightarrow+\infty} x^{k_{j}}=\hat{x}$. Probaremos que $\hat{x} \in E$.

Dado que por $(\mathbf{H 2}) F$ es localmente Lipschitz en $\mathbb{R}^{n}$, entonces la función $\langle F(), z$.$\rangle también$ es localmente Lipschitz, y por lo tanto continua en $\mathbb{R}^{n}$ para todo $z \in \mathbb{R}^{p}$, en particular para $z \in \mathbb{R}_{\geqq}^{p} \backslash\{0\}$; así que $\operatorname{lím}_{j \rightarrow+\infty}\left\langle F\left(x^{k_{j}}\right), z\right\rangle=\langle F(\hat{x}), z\rangle$. Por otro lado, como $x^{k+1} \in \Omega_{k}$ (lo cual se demostró en el Teorema 3.2.1), entones $F_{i}\left(x^{k+1}\right) \leq F_{i}\left(x^{k}\right)$ para todo $i \in\{1, \cdots, p\}$, y como $z \in \mathbb{R}_{\geqq}^{p} \backslash\{0\}$, entonces $\left\langle F\left(x^{k+1}\right), z\right\rangle \leq\left\langle F\left(x^{k}\right), z\right\rangle$. Dado que la función $\langle F(), z$.$\rangle es decreciente, y$ además se encuentra acotada inferiormente ${ }^{3}$, entonces la sucesión $\left\{\left\langle F\left(x^{k}\right), z\right\rangle\right\}$ es convergente. Así que

$$
\langle F(\hat{x}), z\rangle=\lim _{k \rightarrow+\infty}\left\langle F\left(x^{k}\right), z\right\rangle:=\inf _{k \in \mathbb{N}}\left\{\left\langle F\left(x^{k}\right), z\right\rangle\right\} \leq\left\langle F\left(x^{k}\right), z\right\rangle .
$$

\footnotetext{
${ }^{2}$ Por el Teorema 3.2.1 la sucesión $\left\{x^{k+1}\right\}$ generada por el MPPEI existe, y satisface $F\left(x^{k+1}\right) \preceq F\left(x^{k}\right)$ para todo $k \in \mathbb{N}$; entonces aplicando la hipótesis (H4), existe $a \in \mathbb{R}^{n}$ tal que $F(a) \preceq F\left(x^{k}\right)$, así que $a \in E$.

${ }^{3}$ Dado que $0 \preceq F(\operatorname{ver}(\mathbf{H 1}))$ y $z \in \mathbb{R}_{\geqq}^{p} \backslash\{0\}$, entonces $0 \leq\langle F($.), $z\rangle$.
} 
De la expresión anterior se tiene que $\left\langle F\left(x^{k}\right)-F(\hat{x}), z\right\rangle \geq 0 \forall k \in \mathbb{N}$ y $\forall z \in \mathbb{R}_{\geqq}^{p} \backslash\{0\}$, lo cual implica que $F\left(x^{k}\right)-F(\hat{x}) \in \mathbb{R}_{\geqq}^{p}$, es $\operatorname{decir} F(\hat{x}) \preceq F\left(x^{k}\right)$ para todo $k \in \mathbb{N}$, así que $\hat{x} \in E$.

Proposición 3.3.1 Sean $\left\{x^{k}\right\}$ y $\left\{e^{k}\right\}$ las sucesiones generadas por el MPPEI. Si las hipótesis (H1), (H2), (H3) y (H4) son satisfechas, entonces para todo $x \in E$ y para todo $k \in \mathbb{Z}_{0}^{+}$se tiene que

$$
\left\|x^{k+1}-x\right\|^{2} \leq\left\|x^{k}-x\right\|^{2}-\left\|x^{k+1}-x^{k}\right\|^{2}-\frac{2}{\alpha_{k}}\left\langle e^{k+1}, x-x^{k+1}\right\rangle .
$$

Demostración. Por el Teorema 3.2.1, sabemos que la sucesión $\left\{x^{k}\right\}$ generada por el algoritmo MPPEI, existe. Entonces aplicando a la relación (8) el Lema 2.2.4, se tiene que

$$
e^{k+1} \in \partial^{0}\left(\sum_{i=1}^{p} z_{k}^{i} F_{i}(.)\right)\left(x^{k+1}\right)+\alpha_{k}\left(x^{k+1}-x^{k}\right)+N_{\Omega_{k}}\left(x^{k+1}\right) .
$$

Aplicando también los Lemas 2.2.3 y 2.2.2, a uno de los términos de la expresión anterior, tenemos

$$
\partial^{0}\left(\sum_{i=1}^{p} z_{k}^{i} F_{i}(.)\right)\left(x^{k+1}\right) \subset \sum_{i=1}^{p} \partial^{0}\left(z_{k}^{i} F_{i}\left(x^{k+1}\right)\right)=\sum_{i=1}^{p} z_{k}^{i} \partial^{0} F_{i}\left(x^{k+1}\right) .
$$

Reemplazando la expresión anterior en (11), y despejando el término $x^{k}-x^{k+1}$, se tiene que

$$
x^{k}-x^{k+1}=\frac{1}{\alpha_{k}}\left(\sum_{i=1}^{p} z_{k}^{i} g_{i}^{k}+v_{k}-e^{k+1}\right),
$$

donde $g_{i}^{k} \in \partial^{0} F_{i}\left(x^{k+1}\right)$ y $v_{k} \in N_{\Omega_{k}}\left(x^{k+1}\right)$. Ahora tomemos un punto arbitrario $\bar{x} \in E$. Por definición de $\mathrm{E}(\operatorname{ver}(9))$ se tiene que $\bar{x} \in \Omega_{k}$ para todo $k$, en particular $\bar{x} \in \Omega_{k+1}$. Como $\mathrm{E}$ es un conjunto cerrado no vacío, int $\Omega_{k+1} \neq \emptyset$, así que existe $\left\{x^{l}\right\} \subset$ int $\Omega_{k+1}$ tal que $x^{l} \rightarrow \bar{x}$. Por otro lado es claro que

$$
\left\|x^{k}-x\right\|^{2}=\left\|x^{k}-x^{k+1}\right\|^{2}+\left\|x^{k+1}-x\right\|^{2}+2\left\langle x^{k}-x^{k+1}, x^{k+1}-x\right\rangle .
$$

Reemplazando (12) en la expresión anterior y haciendo que $x=x^{l}$ se tiene que

$$
\begin{aligned}
\left\|x^{k}-x^{l}\right\|^{2}= & \left\|x^{k}-x^{k+1}\right\|^{2}+\left\|x^{k+1}-x^{l}\right\|^{2}+ \\
& \frac{2}{\alpha_{k}}\left(\sum_{i=1}^{p} z_{k}^{i}\left\langle g_{i}^{k}, x^{k+1}-x^{l}\right\rangle+\left\langle v_{k}, x^{k+1}-x^{l}\right\rangle-\left\langle e^{k+1}, x^{k+1}-x^{l}\right\rangle\right) .
\end{aligned}
$$

Dado que $F$ es una función propia, cuasi-convexa y localmente Lipschitz, $g_{i}^{k} \in \partial^{0} F_{i}\left(x^{k+1}\right)$, y además $F_{i}\left(x^{l}\right)<F_{i}\left(x^{k+1}\right)$ para todo $i \in\{1, \cdots, p\}$ (esto se deduce del hecho que $x^{l} \in$ int $\Omega_{k+1}$ ), por la Proposición 2.2.6 se tiene que

$$
\left\langle g_{i}^{k}, x^{k+1}-x^{l}\right\rangle \geq 0 \quad \forall i \in\{1, \cdots, p\} .
$$

Por otro lado, dado que $v_{k} \in N_{\Omega_{k}}\left(x^{k+1}\right)$, entonces $\left\langle v_{k}, w-x^{k+1}\right\rangle \leq 0$ para todo $w \in \Omega_{k} \subseteq \Omega_{k+1}$ (ver Definición 2.1.2), en particular para $x^{l} \in \Omega_{k+1}$; entonces

$$
\left\langle v_{k}, x^{k+1}-x^{l}\right\rangle \geq 0 \quad \forall i \in\{1, \cdots, p\} .
$$

Reemplazando las desigualdades (15) y (14) en (13), se tiene que

$$
\left\|x^{k}-x^{l}\right\|^{2} \geq\left\|x^{k}-x^{k+1}\right\|^{2}+\left\|x^{k+1}-x^{l}\right\|^{2}-\frac{2}{\alpha_{k}}\left\langle e^{k+1}, x^{k+1}-x^{l}\right\rangle \quad \forall k \in \mathbb{N} .
$$

Finalmente haciendo que $l \rightarrow+\infty$, tenemos que $\left\|x^{k+1}-\bar{x}\right\|^{2} \leq\left\|x^{k}-\bar{x}\right\|^{2}-\left\|x^{k+1}-x^{k}\right\|^{2}-$ $\frac{2}{\alpha_{k}}\left\langle e^{k+1}, \bar{x}-x^{k+1}\right\rangle$ para todo $k \in \mathbb{N}$, de donde claramente se sigue el resultado. 


\section{Algunas variantes del algoritmo MPPEI para minimización multiobjetivo Quasi-convexa}

En esta sección siguiendo un enfoque semejante al realizado por [4], presentamos y analizamos los resultados de convergencia de dos variantes del algoritmo MPPEI, obtenidas agregando al mismo dos criterios de error, el primero de los cuáles es una versión clásica introducida por [27], y el segundo de ellos, estudiado por [30]. Además, bajo ciertas suposiciones probamos que la sucesión generada por cada una de estas variantes, converge hacia algún punto crítico ParetoClarke del problema (6).

\subsection{Algoritmo MPPEI1 y resultados de convergencia}

En esta subsección discutimos el algoritmo MPPEI, afectado por la versión clásica de criterio de estimación del error (introducida por [27]), a lo que que llamaremos el algoritmo MPPEI1, el cuál está definido por:

Algorithm 2*

Algoritmo MPPEI1 La sucesión $\left\{x^{k}\right\}$ generada por el algoritmo MPPEI (ver las relaciones (7) y (8)), para el término del error $e^{k+1}$ satisface la siguiente hipótesis:

$$
\text { (E1) } \sum_{k=0}^{+\infty} \frac{\left\|e^{k+1}\right\|}{\alpha_{k}}<+\infty
$$

Observación 4.1.1 De acuerdo con la hipótesis (E1), es importante observar que existe un cierto margen para elegir $e^{k+1}$. De hecho, esta suposición fue considerada natural en el algoritmo de punto proximal inexacto clásico introducido por [27].

Lema 4.1.1 Sean $\left\{x^{k}\right\}$ y $\left\{e^{k}\right\}$ las sucesiones generadas por el MPPEI1. Si las hipótesis (H1), (H2), (H3) y (H4) son satisfechas, entonces: (i) la sucesión $\left\{x^{k}\right\}$ es acotada; (ii) $\lim _{k \rightarrow+\infty} \| x^{k+1}$ $x^{k} \|=0, y$ (iii) $\lim _{j \rightarrow+\infty} x^{k_{j}+1}=\hat{x}$, cuando $\lim _{j \rightarrow+\infty} x^{k_{j}}=\hat{x}$.

Demostración. i) Sea $\bar{x} \in E$ un punto fijo. Por la Proposición 3.3.1, y la aplicación de la desigualdad de Cauchy-Schwarz, se tiene que

$$
\left\|x^{k+1}-\bar{x}\right\|^{2} \leq\left\|x^{k}-\bar{x}\right\|^{2}+2 \frac{\left\|e^{k+1}\right\|}{\alpha_{k}}\left\|x^{k+1}-\bar{x}\right\| .
$$

Dado que $\left(a-\frac{1}{2}\right)^{2}=a^{2}-a+\frac{1}{4} \geq 0$ para todo $a \in \mathbb{R}$, entonces $a \leq a^{2}+\frac{1}{4}$. Considerando en esta última desigualdad que $a=\left\|x^{k+1}-\bar{x}\right\|$, y reemplazando dicho resultado en la expresión anterior, se tiene que

$$
\left(1-2 \frac{\left\|e^{k+1}\right\|}{\alpha_{k}}\right)\left\|x^{k+1}-\bar{x}\right\|^{2} \leq\left\|x^{k}-\bar{x}\right\|^{2}+\frac{1}{2} \frac{\left\|e^{k+1}\right\|}{\alpha_{k}} .
$$

Haciendo que $E^{k}=\frac{\left\|e^{k+1}\right\|}{\alpha_{k}}$, tenemos

$$
\left\|x^{k+1}-\bar{x}\right\|^{2} \leq\left(1+\frac{2 E^{k}}{1-2 E^{k}}\right)\left\|x^{k}-\bar{x}\right\|^{2}+\frac{E^{k}}{2}\left(\frac{1}{1-2 E^{k}}\right) .
$$


Como $E^{k} \rightarrow 0$, entonces existe $k_{0} \in \mathbb{N}$, tal que $E^{k}<1 / 4$ para todo $k \geq k_{0}$, lo cual implica que $\left(\frac{1}{1-2 E^{k}}\right)<2$, y que $\left(1+\frac{2 E^{k}}{1-2 E^{k}}\right)<1+2\left(2 E^{k}\right)$. Reemplazando estas últimas desigualdades en la expresión anterior, tenemos que

$$
\left\|x^{k+1}-\bar{x}\right\|^{2} \leq\left(1+4 E^{k}\right)\left\|x^{k}-\bar{x}\right\|^{2}+E^{k} .
$$

Finalmente haciendo uso del Lema 2.1.1 se tiene que $\left\|x^{k+1}-\bar{x}\right\|^{2}$ converge, dado que en la expresión anterior los términos $v_{k}=\left\|x^{k+1}-\bar{x}\right\|^{2}, v_{k-1}=\left\|x^{k}-\bar{x}\right\|^{2}, \lambda_{k}=4 E^{k}$ y $\beta_{k}=E^{k}$ satisfacen lo siguiente: $\sum_{k=1}^{+\infty} \lambda_{k}<\infty$ y $\sum_{k=1}^{+\infty} \beta_{k}<\infty$. Por lo tanto la sucesión $\left\{x^{k}\right\}$ es acotada.

ii) Aplicando nuevamente la desigualdad de Cauchy-Schwarz a la Proposición 3.3.1, se tiene que

$$
\left\|x^{k+1}-x^{k}\right\|^{2} \leq\left\|x^{k}-\bar{x}\right\|^{2}-\left\|x^{k+1}-\bar{x}\right\|^{2}+2 \frac{\left\|e^{k+1}\right\|}{\alpha_{k}}\left\|x^{k+1}-\bar{x}\right\| .
$$

Aplicando sumatorias, tenemos

$$
\sum_{k=0}^{m}\left\|x^{k+1}-x^{k}\right\|^{2} \leq\left\|x^{0}-\bar{x}\right\|^{2}-\left\|x^{m+1}-\bar{x}\right\|^{2}+2 \max _{0 \leq k \leq m}\left\{\left\|x^{k+1}-\bar{x}\right\|\right\} \sum_{k=0}^{m} \frac{\left\|e^{k+1}\right\|}{\alpha_{k}} .
$$

Haciendo que $m \rightarrow \infty$, y teniendo en cuenta la hipótesis (E1) y el resultado anterior (item (i)) se tiene que $\sum_{k=0}^{+\infty}\left\|x^{k+1}-x^{k}\right\|^{2}<+\infty$, de donde se sigue el resultado.

iii) Es claro que $\left\|x^{k_{j}+1}-\hat{x}\right\| \leq\left\|x^{k_{j}+1}-x^{k_{j}}\right\|+\left\|x^{k_{j}}-\hat{x}\right\|$. Tomando límite cuando $j \rightarrow+\infty, \mathrm{y}$ considerando el ítem (ii) y la hipótesis $\lim _{k \rightarrow+\infty} x^{k_{j}}=\hat{x}$, en la desigualdad anterior se tiene que $\lim _{j \rightarrow+\infty}\left\|x^{k_{j}+1}-\hat{x}\right\| \leq 0$, de donde se sigue el resultado.

Proposición 4.1.1 Sean $\left\{x^{k}\right\}$ y $\left\{e^{k}\right\}$ las sucesiones generadas por el MPPEI1. Si las hipótesis (H1), (H2), (H3) y (H4) son satisfechas, entonces la sucesión $\left\{x^{k}\right\}$ converge a un punto de E.

Demostración. Por el Lema 4.1.1(i) se tiene que la sucesión $\left\{x^{k}\right\}$ es acotada, entonces existe una subsucesión $\left\{x^{k_{j}}\right\} \subseteq\left\{x^{k}\right\}$ y al menos un punto de acumulación $\hat{x} \in \mathbb{R}^{n}$, tales que $x^{k_{j}} \rightarrow \hat{x}$. Así que por el Lema 3.3.1 se tiene que $\hat{x} \in E$, lo cual implica el resultado.

A continuación, considerando la acotación de los parámetros regulares $\left\{\alpha_{k}\right\}$, probaremos que las sucesiones iterantes convergen hacia un punto crítico Pareto-Clarke del problema (6).

Teorema 4.1.1 Si la función $F: \mathbb{R}^{n} \rightarrow \mathbb{R}^{p}$, tal que $F_{i}: \mathbb{R}^{n} \rightarrow \mathbb{R} \cup\{+\infty\}$, satisface las hipótesis (H1), (H2), (H3) y (H4), con $0<\alpha_{k} \leq \bar{\alpha}$, entonces la sucesión $\left\{x^{k}\right\}$ generada por MPPEI1 converge hacia algún punto crítico Pareto-Clarke del POM (6).

Demostración. Por la Proposición 4.1.1 existe $\hat{x} \in E$ tal que $x^{k} \rightarrow \hat{x}$. Probaremos que $\hat{x}$ es un punto crítico Pareto-Clarke (ver Definición 2.4.1). Para llegar a una contradicción asumimos lo contrario, entonces existe algún vector $d \in \mathbb{R}^{n}$, tal que

$$
F_{i}^{0}(\hat{x}, d)<0 \quad \forall i \in\{1, \cdots, p\},
$$

lo cual implica que $d$ es una dirección de descenso para la función multiobjetivo $F$ en $\hat{x}$, así que existe $\delta>0$ tal que $F(\hat{x}+\lambda d)<F(\hat{x})$ for all $\lambda \in(0, \delta]$. Por lo tanto $(\hat{x}+\lambda d) \in \Omega_{k}$. Por otro lado, por el Teorema 3.2.1 conocemos que la sucesión $\left\{x^{k+1}\right\}$ generada por el MPPEI existe, y además $x^{k+1} \in \Omega_{k}$. Así que en (11), se tiene que

$$
\alpha_{k}\left(x^{k}-x^{k+1}\right)-v_{k}+e^{k+1} \in \partial^{0}\left(\left\langle F(.), z_{k}\right\rangle\right)\left(x^{k+1}\right),
$$


donde $v_{k} \in N_{\Omega_{k}}\left(x^{k+1}\right)$. Haciendo uso de la Definición 2.2.2 concerniente al término $\partial^{0}($.$) , en la$ expresión anterior se tiene que $\left\langle\alpha_{k}\left(x^{k}-x^{k+1}\right)-v_{k}+e^{k+1}, q\right\rangle \leq\left\langle F(.), z_{k}\right\rangle^{0}\left(x^{k+1}, q\right)$ para todo $q \in \mathbb{R}^{n}$, donde $\left\langle F(.), z_{k}\right\rangle^{0}\left(x^{k+1}, q\right)$ representa la derivada direccional de Clarke. Utilizando propiedades básicas del producto interno de vectores, definiendo $q=\left(\hat{x}+\lambda d-x^{k+1}\right)$, y considerando que $v_{k} \in N_{\Omega_{k}}\left(x^{k+1}\right)$, en la desigualdad anterior se tiene que

$$
\alpha_{k}\left\langle x^{k}-x^{k+1}, q\right\rangle+\left\langle e^{k+1}, q\right\rangle \leq\left\langle F(.), z_{k}\right\rangle^{0}\left(x^{k+1}, q\right)=\left(\sum_{i=1}^{p} z_{k}^{i} F_{i}(.)\right)^{0}\left(x^{k+1}, q\right) .
$$

Aplicando respectivamente los ítems (i), (ii) y (iii) del Lema 2.2.1, al término de la derecha de la desigualdad anterior, se tiene

$$
\left(\sum_{i=1}^{p} z_{k}^{i} F_{i}(.)\right)^{0}\left(x^{k+1}, q\right) \leq \sum_{i=1}^{p}\left(z_{k}^{i} F_{i}\right)^{0}\left(x^{k+1}, q\right)=\sum_{i=1}^{p} z_{k}^{i} F_{i}^{0}\left(x^{k+1}, q\right)=\sum_{i=1}^{p} F_{i}^{0}\left(x^{k+1}, z_{k}^{i} q\right),
$$

donde $z_{k}^{i}$ son las componentes del vector $z_{k}$. Reemplazando la expresión anterior en (17), y aplicando la desigualdad de Cauchy Schwarz, así como también algunas propiedades básicas relacionadas al producto interno, y a la norma vectorial, se tiene que

$$
-\alpha_{k}\left\|x^{k}-x^{k+1}\right\|\|q\|-\alpha_{k}\left(\frac{\left\|e^{k+1}\right\|}{\alpha_{k}}\right)\|q\| \leq \sum_{i=1}^{p} F_{i}^{0}\left(x^{k+1}, z_{k}^{i} q\right) .
$$

Como $\left\{z_{k}\right\}$ es acotada, entonces existe una subsucesión $\left\{z_{k_{j}}\right\}$ tal que $l i m_{j \rightarrow+\infty} z_{k_{j}}=\bar{z} \in \mathbb{R}_{\geqq}^{p} \backslash\{0\}$. De ser necesario, y sin perdida de generalidad, también podemos considerar las subsucesiones $\left\{x^{k_{j}}\right\} \subseteq\left\{x^{k}\right\}$ y $\left\{\alpha_{k_{j}}\right\} \subseteq\left\{\alpha_{k}\right\}$. Además, como $0<\alpha_{k} \leq \bar{\alpha}$, en (18) se tiene que

$$
-\bar{\alpha}\left\|x^{k_{j}}-x^{k_{j}+1}\right\|\left\|q^{\prime}\right\|-\bar{\alpha}\left(\frac{\left\|e^{k_{j}+1}\right\|}{\alpha_{k_{j}}}\right)\left\|q^{\prime}\right\| \leq \sum_{i=1}^{p} F_{i}^{0}\left(x^{k_{j}+1}, z_{k_{j}}^{i} q^{\prime}\right),
$$

donde $q^{\prime}=\left(\hat{x}+\lambda d-x^{k_{j}+1}\right)$, y además $z_{k_{j}}^{i}$ son las componentes del vector $z_{k_{j}}$. Tomando limite superior cuando $j \rightarrow+\infty$, y considerando los ítems (ii) y (iii) del Lema 4.1.1, la hipótesis (E1), y el hecho que $\lambda>0$ y $z_{k_{j}} \rightarrow \bar{z}$, se puede verificar que el término de la izquierda de la desigualdad anterior tiende a cero. Entonces en (19) tenemos que:

$$
0 \leq \limsup _{j \rightarrow+\infty} \sum_{i=1}^{p} F_{i}^{0}\left(x^{k_{j}+1}, z_{k_{j}}^{i} q^{\prime}\right)=\sum_{i=1}^{p} \limsup _{j \rightarrow+\infty} F_{i}^{0}\left(x^{k_{j}+1}, z_{k_{j}}^{i} q^{\prime}\right)
$$

Como $F$ es propia y localmente Lipschitz, entonces aplicando la Proposición 2.2.5 se tiene que

$$
0 \leq \sum_{i=1}^{p} \limsup _{j \rightarrow+\infty} F_{i}^{0}\left(x^{k_{j}+1}, z_{k_{j}}^{i} q^{\prime}\right) \leq \sum_{i=1}^{p} F_{i}^{0}\left(\hat{x}, \bar{z}^{i} \lambda d\right) .
$$

Haciendo uso del Lema 2.2.1(iii), y considerando el hecho que $\lambda>0$, en (20) se tiene que

$$
\sum_{i=1}^{p} \bar{z}^{i} F_{i}^{0}(\hat{x}, d)=\bar{z}^{1} F_{1}^{0}(\hat{x}, d)+\cdots+\bar{z}^{p} F_{p}^{0}(\hat{x}, d) \geq 0
$$

Como $\bar{z} \in \mathbb{R}_{\geqq}^{p} \backslash\{0\}$, entonces sin pérdida de generalidad consideramos $I=\left\{i \in\{1, \cdots, p\}: \bar{z}^{i}>\right.$ $0\}$. Así que en (21) existe algún $i_{0} \in I$ tal que $F_{i_{0}}^{0}(\hat{x}, d) \geq 0$, lo cual contradice (16). Por lo tanto $\hat{x}$ es un punto crítico Pareto-Clarke del problema (6). 
Corolario 4.1.1 Sea $F: \mathbb{R}^{n} \rightarrow \mathbb{R}^{p}$ una función $\mathbb{R}_{\geqq}^{p}$-convexa que satisface las hipótesis (H1), (H2), (H3) y (H4), con $0<\alpha_{k} \leq \bar{\alpha}$. Si $\hat{x}$ es un punto de convergencia de la sucesión $\left\{x^{k}\right\}$ generada por algoritmo MPPEI1, entonces $\hat{x}$ es una solución débil de Pareto del POM (6).

Demostración. Como $\hat{x}$ es un punto de convergencia de $\left\{x^{k}\right\}$, por el Teorema 4.1.1 $\hat{x}$ es un punto crítico Pareto-Clarke. Finalmente haciendo uso de la Proposición 2.4.1 se tiene que $\hat{x}$ es una solución débil de Pareto del POM (6).

Corolario 4.1.2 Si $F: \mathbb{R}^{n} \rightarrow \mathbb{R}^{p}$ es una función continuamente diferenciable en $\mathbb{R}^{n}$, y además satisface las hipótesis $(\boldsymbol{H} 1),(\boldsymbol{H} 3)$ y $(\boldsymbol{H} 4)$, con $0<\alpha_{k} \leq \bar{\alpha}$, entonces el punto de convergencia $\hat{x}$ de la sucesión $\left\{x^{k}\right\}$ generada por el MPPEI1, es un punto crítico de Pareto-Clarke del problema (6), es decir, existe $i_{0} \in\{1,2, \cdots, p\}$ tal que

$$
\left\langle\nabla F_{i_{0}}(\hat{x}), d\right\rangle \geq 0 \quad \forall d \in \mathbb{R}^{n} .
$$

Demostración. Es inmediata, dado que continuamente diferenciable en $\mathbb{R}^{n}$ implica la suposición (H2). Así que por el Teorema 2.2.1 tenemos que $F_{i}^{0}(\hat{x}, d)=F_{i}^{\prime}(\hat{x}, d)=\left\langle\nabla F_{i}(\hat{x}), d\right\rangle$, donde $F_{i}^{\prime}$ es la derivada direccional de $F_{i}$. Finalmente aplicando el Teorema 4.1.1 obtenemos el resultado deseado.

\subsection{Algoritmo MPPEI2 y convergencia de resultados}

En esta subsección discutimos el algoritmo MPPEI, afectado por el criterio de de error (introducido por [30]), a lo que que llamaremos el algoritmo MPPEI2, el cuál está definido por:

Algorithm 3*

Algoritmo MPPEI2 La sucesión $\left\{x^{k}\right\}$ generada por el algoritmo MPPEI (ver las relaciones (7) y (8)), para el término del error $e^{k+1}$ satisface las siguientes hipótesis:

(E2)

$$
\begin{aligned}
\text { (E2) } \frac{\left\|e^{k+1}\right\|}{\alpha_{k}} \leq \eta_{k}\left\|x^{k+1}-x^{k}\right\| \\
\text { (E2.1) } \sum_{k=0}^{+\infty} \eta_{k}^{2}<+\infty
\end{aligned}
$$

Lema 4.2.1 Sean $\left\{x^{k}\right\}$ y $\left\{e^{k}\right\}$ las sucesiones generadas por el MPPEI2. Si las hipótesis (H1), (H2), (H3) y (H4) son satisfechas, entonces existe un entero $k_{0} \geq 0$, tal que para todo $k \geq k_{0}$ se tiene que

$$
\left\|x^{k+1}-x\right\|^{2} \leq\left(1+\frac{2 \eta_{k}^{2}}{1-2 \eta_{k}^{2}}\right)\left\|x^{k}-x\right\|^{2}-\frac{1}{2}\left\|x^{k+1}-x^{k}\right\|^{2} \quad \forall x \in E .
$$

Además, la sucesión $\left\{x^{k}\right\}$ es acotada, $\lim _{k \rightarrow+\infty}\left\|x^{k+1}-x^{k}\right\|=0, y \lim _{j \rightarrow+\infty} x^{k_{j}+1}=x \in E$, cuando $\lim _{j \rightarrow+\infty} x^{k_{j}}=x$.

Demostración. i) Probaremos (23). Es claro que $\langle y, y\rangle \geq 0$ para todo $y \in \mathbb{R}^{n}$; haciendo $y=\frac{e^{k+1}}{\sqrt{2 \alpha_{k}} \eta_{k}}-\sqrt{2 \alpha_{k}} \eta_{k}\left(x^{k+1}-x\right)$, y considerando el hecho que $\eta_{k} \rightarrow 0^{+}$, tenemos que

$$
0 \leq\langle y, y\rangle=\frac{1}{2 \alpha_{k} \eta_{k}^{2}}\left\|e^{k+1}\right\|^{2}-2\left\langle e^{k+1}, x^{k+1}-x\right\rangle+2 \alpha_{k} \eta_{k}^{2}\left\|x^{k+1}-x\right\|^{2},
$$


de donde

$$
-2\left\langle e^{k+1}, x-x^{k+1}\right\rangle \leq \frac{1}{2 \alpha_{k} \eta_{k}^{2}}\left\|e^{k+1}\right\|^{2}+2 \alpha_{k} \eta_{k}^{2}\left\|x^{k+1}-x\right\|^{2} .
$$

Reemplazando (24) en (10), y haciendo uso de la hipótesis (E2) tenemos que

$$
\left(1-2 \eta_{k}^{2}\right)\left\|x^{k+1}-x\right\|^{2} \leq\left\|x^{k}-x\right\|^{2}-\frac{1}{2}\left\|x^{k+1}-x^{k}\right\|^{2} .
$$

Como $\eta_{k} \rightarrow 0^{+}$, lo cual se cumple por la condición (E2.1), entonces existe $k_{0} \geq 0$ tal que $0<1-2 \eta_{k}^{2}<1$ para todo $k \geq k_{0}$. Así que en la expresión anterior

$$
\left\|x^{k+1}-x\right\|^{2} \leq\left(1+\frac{2 \eta_{k}^{2}}{1-2 \eta_{k}^{2}}\right)\left\|x^{k}-x\right\|^{2}-\frac{1}{2}\left\|x^{k+1}-x^{k}\right\|^{2},
$$

con lo cual obtenemos la primera parte del teorema.

ii) Probaremos que $\left\{x^{k}\right\}$ es una sucesión acotada. De la desigualdad (23), es claro que

$$
\left\|x^{k+1}-x\right\|^{2} \leq\left(1+\frac{2 \eta_{k}^{2}}{1-2 \eta_{k}^{2}}\right)\left\|x^{k}-x\right\|^{2} \quad \forall k \geq k_{0} .
$$

Dado que $\eta_{k}^{2} \rightarrow 0^{+}$, para todo $\epsilon>0$ existe $\widetilde{k_{0}} \geq 0$, tal que $\eta_{k}^{2}<\epsilon$ para todo $k \geq \widetilde{k_{0}}$. Entonces $0<1-2 \epsilon<1-2 \eta_{k}^{2}<1$. Por lo tanto para todo $\epsilon<1 / 2$, y para todo $k \geq k_{0}$ se tiene que

$$
\frac{2 \eta_{k}^{2}}{1-2 \eta_{k}^{2}}<\frac{2 \eta_{k}^{2}}{1-2 \epsilon} \quad \forall k \geq \widetilde{k_{0}}
$$

Aplicando sumatorias, y teniendo en cuenta la hipótesis (E2.1), se tiene que

$$
\sum_{k=1}^{+\infty} \frac{2 \eta_{k}^{2}}{1-2 \eta_{k}^{2}}<+\infty
$$

Finalmente haciendo uso del Lema 2.1.1 se tiene que $\left\|x^{k+1}-x\right\|^{2}$ converge, dado que en la expresión (25) los términos $v_{k}=\left\|x^{k+1}-x\right\|^{2}, v_{k-1}=\left\|x^{k}-x\right\|^{2}, \lambda_{k}=\frac{2 \eta_{k}^{2}}{1-2 \eta_{k}^{2}}$ y $\beta_{k}=0$, satisfacen $\sum_{k=1}^{+\infty} \lambda_{k}<\infty$ (ver la relación (26)) y $\sum_{k=1}^{+\infty} \beta_{k}<\infty$. Por lo tanto la sucesión $\left\{x^{k}\right\}$ es acotada.

iii) Probaremos que $\lim _{k \rightarrow+\infty}\left\|x^{k+1}-x^{k}\right\|=0$. Por la relación (23) es claro que

$$
\frac{1}{2}\left\|x^{k+1}-x^{k}\right\| \leq\left\|x^{k}-x\right\|^{2}-\left\|x^{k+1}-x\right\|^{2}+\frac{2 \eta_{k}^{2}}{1-2 \eta_{k}^{2}}\left\|x^{k}-x\right\|^{2} .
$$

Aplicando sumatorias se tiene que

$$
\frac{1}{2} \sum_{k=k_{0}}^{m}\left\|x^{k+1}-x^{k}\right\| \leq\left\|x^{k_{0}}-x\right\|^{2}-\left\|x^{m+1}-x\right\|^{2}+\max _{k_{0} \leq k \leq m}\left\{\left\|x^{k}-x\right\|^{2}\right\} \sum_{k=k_{0}}^{m} \frac{2 \eta_{k}^{2}}{1-2 \eta_{k}^{2}} .
$$

Dado que $\left\{x^{k}\right\}$ es una sucesión acotada, $\left\|x^{k}-x\right\|$ para todo $k \in \mathbb{N}$ también es acotada. Entonces tomando límite cuando $m \rightarrow+\infty$, y considerando la expresión (26), tenemos que $\sum_{k=k_{0}}^{+\infty}\left\|x^{k+1}-x^{k}\right\|<+\infty$; así que $\lim _{k \rightarrow+\infty}\left\|x^{k+1}-x^{k}\right\|=0$.

vi) La demostración pendiente es semejante a la prueba realizada en el Lema 4.1.1 - ítem (iii). 
Proposición 4.2.1 Sean $\left\{x^{k}\right\}$ y $\left\{e^{k}\right\}$ las sucesiones generadas por el algoritmo MPPEI2. Si las hipótesis (H1), (H2), (H3) y (H4) son satisfechas, entonces la sucesión $\left\{x^{k}\right\}$ converge a un punto de E.

Demostración. Por el Lema 4.2.1 se tiene que la sucesión $\left\{x^{k}\right\}$ es acotada, entonces existe al menos un punto de acumulación $\hat{x} \in \mathbb{R}^{n}$, así como también una subsucesión $\left\{x^{k_{j}}\right\} \subseteq\left\{x^{k}\right\}$, tales que $x^{k_{j}} \rightarrow \hat{x}$. Entonces por el Lema 3.3.1 se tiene que $\hat{x} \in E$, lo cual implica el resultado.

Teorema 4.2.1 Si la función $F: \mathbb{R}^{n} \rightarrow \mathbb{R}^{p}$ satisface las hipótesis (H1), (H2), (H3) y (H4), con $0<\alpha_{k} \leq \bar{\alpha}$, entonces la sucesión $\left\{x^{k}\right\}$ generada por el MPPEI2 converge hacia algún punto crítico Pareto-Clarke del POM (6).

Demostración. Considerando que $\left\{x^{k}\right\}$ es la sucesión generada por el algoritmo MPPEI2, tomamos un $\hat{x} \in \mathbb{R}^{n}$ tal que $x^{k} \rightarrow \hat{x}$. Para probar que $\hat{x}$ es un punto crítico Pareto Clarke, suponemos lo contrario; entonces siguiendo los mismos pasos realizados de la primera parte de la prueba del Teorema 4.1.1, hasta la relación (18), se tiene que

$$
-\alpha_{k}\left\|x^{k}-x^{k+1}\right\|\|q\|-\left\|e^{k+1}\right\|\|q\| \leq \sum_{i=1}^{p} F_{i}^{0}\left(x^{k+1}, z_{k}^{i} q\right) .
$$

Reemplazando (E2), y teniendo en cuenta que $0<\alpha_{k} \leq \bar{\alpha}$, en la expresión anterior se tiene que

$$
\begin{aligned}
-\alpha_{k}\left\|x^{k}-x^{k+1}\right\|\|q\|-\alpha_{k} \eta_{k}\left\|x^{k+1}-x^{k}\right\|\|q\| & \leq \sum_{i=1}^{p} F_{i}^{0}\left(x^{k+1}, z_{k}^{i} q\right) \\
-\bar{\alpha}(1+M)\left\|x^{k+1}-x^{k}\right\|\|q\| & \leq \sum_{i=1}^{p} F_{i}^{0}\left(x^{k+1}, z_{k}^{i} q\right)
\end{aligned}
$$

para algún $M>0$ (ojo, por la hipótesis $(\mathbf{E 2 . 1}) \eta_{k} \rightarrow 0^{+}$). Siguiendo nuevamente los pasos que se encuentran entre las relaciones (18) y (19) del Teorema 4.1.1, tenemos que

$$
-\bar{\alpha}(1+M)\left\|x^{k_{j}+1}-x^{k_{j}}\right\|\left\|q^{\prime}\right\| \leq \sum_{i=1}^{p} F_{i}^{0}\left(x^{k_{j}+1}, z_{k_{j}}^{i} q^{\prime}\right),
$$

donde $q^{\prime}=\left(\hat{x}+\lambda d-x^{k_{j}+1}\right)$. Tomando límite superior cuando $j \rightarrow+\infty$, y siguiendo lo indicado entre las relaciones (19) y (21), considerando en este caso el Lema 4.2.1, en lugar del Lema 4.1.1, se tiene que

$$
0 \leq \sum_{i=1}^{p} \bar{z}^{i} F_{i}^{0}(\hat{x}, d)=\bar{z}^{1} F_{1}^{0}(\hat{x}, d)+\cdots+\bar{z}^{p} F_{p}^{0}(\hat{x}, d),
$$

lo cual representa la misma expresión obtenida en la relación (21). Finalmente siguiendo lo indicado en el párrafo posterior a (21), se tiene que $\hat{x}$ es un punto crítico Pareto-Clarke del problema (6).

Observación 4.2.1 Siguiendo procedimientos semejantes a los desarrollados en los Corolarios 4.1.1 y 4.1.2 (pero considerando ahora el Teorema 4.2.1, en lugar del Teorema 4.1.1), podemos obtener resultados semejantes a los planteados en dichos corolarios. 


\section{Conclusiones y futuras investigaciones}

El aporte del presente trabajo es de carácter teórico, pero con motivación a las aplicaciones. Los resultados de convergencia obtenidos por las dos versiones del algoritmo MPPEI, presentadas en la sección 3, son novedosos en el espacio Euclidiano (incluso para el caso convexo el algoritmo inexacto es novedoso), y aún no han sido publicados por ninguna revista nacional o internacional. Entre los resultados más relevantes, destacamos los siguientes:

1. Presentamos un Método de Punto Proximal Escalarizado Inexacto (MPPEI) para minimización de funciones multiobjetivo cuasi-convexas, definidas en espacios Euclidianos. Además, siguiendo un enfoque semejante al realizado por [4], presentamos dos variantes inexactas del mismo: el MPPEI1 y MPPEI2, cada una de las cuáles es obtenida agregando al MPPEI un criterio de error.

2. Establecemos que la sucesión $\left\{x^{k}\right\}$ generada por el MPPEI está bien definida, y bajo hipótesis razonables (como la acotación superior del parámetro $\alpha_{k}$ ), probamos que la sucesión $\left\{x^{k}\right\}$ generada por cada una de las variantes del MPPEI, converge hacia algún punto crítico Pareto-Clarke del problema de optimización multiobjetivo (6). Además, asumiendo la $\mathbb{R}_{\geq}^{p}$-convexidad de $F$, se prueba que el punto de convergencia de la sucesión $\left\{x^{k}\right\}$, es una solución débil de Pareto del problema (6).

3. Se espera que este artículo sirva como referencia para futuros trabajos de investigación, pues hay otros temas por explorar, tanto en el aspecto teórico como computacional, destacamos los siguientes:

- Puesto que en este artículo solamente estamos interesados en la convergencia teórica del algoritmo inexacto, en un futuro trabajo esperamos realizar su implementación computacional para algunos problemas realistas, y su respectiva comparación con otros métodos. En este punto cabe resaltar que la implementación que presenta [1] de alguna manera ya es inexacta, dado que al resolver los subproblemas generados por el algoritmo, en cada iteración se tiene un error de aproximación.

- La extensión de dicho estudio, hacia el análisis de problemas de minimización multiobjetivo restrictos, los cuáles presentan un mayor rango de aplicación.

\section{Agradecimientos}

Agradecemos a los revisores del artículo que han contribuido con la mejora del trabajo inicial.

\section{Referencias bibliográficas}

[1] Apolinario, H., Papa Quiroz, E. A., y Oliveira, P. R. (2016). A scalarization proximal point method for quasiconvex multiobjective minimization. Journal of Global Optimization, 64 (1), 79-96.

[2] Aussel, D. (1998). Subdiferential properties of quasiconvex and pseudoconvex functions: unified approach. Journal of optimization theory and applications, 97 (1), 29-45.

[3] Baygorrea, N. (2017). Método do ponto proximal inexato para minimização quase-convexa em variedades de Hadamard (Tesis Doctoral). Universidade Federal do Rio de Janeiro, Brasil. 
[4] Baygorrea, N., Papa Quiroz, E. A., y Maculan, N. (2016). Inexact proximal point methods for quasiconvex minimization on Hadamard manifolds. Journal of the Operations Research Society of China, 4 (4), 397-424.

[5] Bazan, F. (2011). Optimización lineal: una mirada introductoria. Santiago de Chile, Chile: Universidad Andres Bello.

[6] Bento, G., Ferreira, O., y Oliveira, P. (2010). Local convergence of the proximal point method for a special class of nonconvex functions on Hadamard manifolds. Nonlinear Analysis: Theory, Methods \& Applications, 73 (2), 564-572.

[7] Berkovitz, L. (2003). Convexity and optimization in $\mathbb{R}^{n}$ (Vol. 63). New York, United States of America: John Wiley \& Sons.

[8] Bolte, J., Daniilidis, A., Lewis, A., y Shiota, M. (2007). Clarke subgradients of strati able functions. SIAM Journal on Optimization, 18 (2), 556-572.

[9] Bonnel, H., Iusem, A., y Svaiter, B. (2005). Proximal methods in vector optimization. SIAM Journal on Optimization, 15 (4), 953-970.

[10] Ceng, L.-C., y Yao, J.-C. (2007). Approximate proximal methods in vector optimization. European Journal of Operational Research, 183 (1), 1-19.

[11] Chen, J.-S., y Pan, S. (2008). A proximal-like algorithm for a class of nonconvex programming. Pacic Journal of Optimization, 4 (2), 319-333.

[12] Clarke, F. (1990). Optimization and nonsmooth analysis (Vol. 5). Philadelphia, United States of America: SIAM.

[13] Clarke, F. (2013). Functional analysis, calculus of variations and optimal control (Vol. 264). New York, United States of America: Springer Science \& Business Media.

[14] Cunha, F., da Cruz Neto, J., y Oliveira, P. R. (2010). A proximal point algorithm with a $\phi$-divergence for quasiconvex programming. Optimization, 59 (5), 777-792.

[15] Custódio, A., Madeira, J., Vaz, A., y Vicente, L. (2011). Direct multisearch for multiobjective optimization. SIAM Journal on Optimization, 21 (3), 1109-1140.

[16] dos Santos Gromicho, J. (2013). Quasiconvex optimization and location theory (Vol. 9). Dordrecht, Holanda: Springer Science \& Business Media.

[17] Ehrgott, M. (2005). Multicriteria optimization (2a. ed.). New York, United States of America: Springer.

[18] Goudou, X., y Munier, J. (2009). The gradient and heavy ball with friction dynamical systems: the quasiconvex case. Mathematical Programming, 116 (1-2), 173-191.

[19] Mas-Colell, A., Whinston, M., y Green, J. (1995). Microeconomic theory (Vol. 1). New York, United States of America: Oxford university press.

[20] Miettinen, K. (2012). Nonlinear multiobjective optimization (Vol. 12). New York, United States of America: Springer Science \&Business Media.

[21] Mordukhovich, B. (2006). Variational analysis and generalized differentiation I: Basic theory (Vol. 330). Berlin, Alemania: Springer Science \& Business Media. 
[22] Pan, S., y Chen, J.-S. (2007). Entropy-like proximal algorithms based on a second-order homogeneous distance function for quasi-convex programming. Journal of Global Optimization, 39 (4), 555-575.

[23] Papa Quiroz, E. A. (2017). Optimización continua: teoría y ejercicios. Madrid, España: Editorial Académica Española.

[24] Papa Quiroz, E. A., Mallma Ramirez, L., y Oliveira, P. R. (2015). An inexact proximal method for quasiconvex minimization. European Journal of Operational Research, 246 (3), 721-729.

[25] Papa Quiroz, E. A., y Oliveira, P. R. (2012). Proximal point method for minimizing quasiconvex locally Lipschitz functions on Hadamard manifolds. Nonlinear Analysis: Theory, Methods \& Applications, 75 (15), 5924-5932.

[26] Polyak, B. (1987). Introduction to optimization. New York, United States of America: Translations Series in Mathematics and Engineering - Springer.

[27] Rockafellar, R. (1976). Monotone operators and the proximal point algorithm. SIAM journal on control and optimization (14), 877-898.

[28] Rockafellar, R., y Wets, R. (2009). Variational analysis (Vol. 317). Berlin, Alemania: Springer Science \& Business Media.

[29] Souza, S., Oliveira, P. R., da Cruz Neto, J., y Soubeyran, A. (2010). A proximal method with separable bregman distances for quasiconvex minimization over the nonnegative orthant. European Journal of Operational Research, 201 (2), 365-376.

[30] Tang, G.-j., y Huang, N.-j. (2013). An inexact proximal point algorithm for maximal monotone vector fields on Hadamard manifolds. Operations Research Letters, 41 (6), 586-591.

[31] Van Tiel, J. (1984). Convex analysis: An introductory text. Chichester, United Kingdom: John Wiley \& Sons.

[32] Villacorta, K., y Oliveira, P. R. (2011). An interior proximal method in vector optimization. European Journal of Operational Research, 214 (3), 485-492. 